\title{
RACIONALIDADES, CREENCIAS Y PRÁCTICAS PEDAGÓGICAS EN LA IMPLEMENTACIÓN DEL CURRÍCULO ESCOLAR
}

\author{
RATIONALITIES, BELIEFS AND \\ PEDAGOGICAL PRACTICES IN THE \\ IMPLEMENTATION OF THE SCHOOL \\ CURRICULUM
}

Juan Luis Condori Gutiérrez

xsidarta@gmail.com

\begin{abstract}
RESUMEN
El presente artículo propone desarrollar una reflexión acerca del tipo de racionalidad que se presenta en la implementación del currículo escolar y de sus relaciones con las creencias y la práctica pedagógica del docente. Consideramos que la racionalidad dominante en el currículo es aquella vinculada con el interés técnico que prioriza la consecución de un producto en relación con unos objetivos preestablecidos que son los estándares de aprendizaje. Asimismo, en las prácticas pedagógicas subyacen rezagos de creencias relacionadas con el cumplimiento de normativas reforzando la idea de que el currículo debe desarrollarse de manera rígida, minimizando las prácticas de construcción curricular desde la experiencia del docente. Por último, el alto nivel prescriptivo establecido desde sus lineamientos para su implementación presenta una serie de elementos que la constituyen como una prescripción más que como una herramienta pertinente con la escuela.
\end{abstract}

\section{PALABRAS CLAVE}

Teorías implícitas, práctica pedagógica, racionalidad tecnicista, implementación curricular.

\section{ABSTRACT}

This article proposes to develop a reflection about the type of rationality presents in the implementation of the school curriculum, and in its relationships with beliefs and pedagogical practice of the teacher. Let us consider that the dominant rationality in the curriculum is associated with technical interest that gives priority to the achievement of a product in relation to pre-established objectives, which are the learning standards. Furthermore, in the pedagogical practices underlie lag of beliefs related to the compliance of regulations reinforcing the idea that the curriculum should be developed in a rigid way, minimizing the practices of curricular construction from the experience of the teacher. Finally, the high prescriptive level established from its guidelines for its implementation presents a series of elements that constitute it as a prescription rather than as a relevant tool with the school.

KEYWORDS

Implicit theories, pedagogical practice, technical rationality, curricular implementation.
$\mathrm{L}$

a política curricular ha devenido en marchas y contramarchas durante el proceso de desarrollo de las reformas educativas en el país. Entre los avances recientes surgidos a partir de la discusión técnica especializada ha sido la reconceptualización del enfoque por competencias, introduciendo en su comprensión la idea de la funcionalidad integral donde la combinación de los recursos debe cumplir el criterio de eficacia para solucionar problemas reales o simulados. También, se ha determinado la evolución de los aprendizajes en un modelo de estándares mínimos que en niveles y ciclos se irán desarrollando de manera progresiva para el logro de las competencias; y se ha insertado el concepto de Perfil de egreso como expresión fundamental de las intenciones del sistema educativo, orientadas al desarrollo personal, a la formación ciudadana y a la preparación para el desempeño laboral.

Sin embargo, en estos vaivenes del desarrollo de la política educativa, las prácticas pedagógicas se han mantenido en la tendencia de continuar con esa subyacente manera de entender el currículo como un documento prescriptivo y disciplinar, moldeable, con ciertas innovaciones de tendencia que terminan por insertarse en la 
didáctica especializada. Como señala Gonzáles (2016), "pareciera que la teoría curricular predominante en los docentes de nuestro país (...) sigue siendo directa, reproductiva $\mathrm{y}$ academicista (...)" (p. 47).

A pesar de los esfuerzos denodados por parte del Estado para ejecutar programas de fortalecimiento profesional, las prácticas de la mayoría de los docentes terminan por alejarse de la propuesta que se enmarca en el currículo por competencias, reforzándose ciertas creencias del cómo debe entenderse el currículo desde unas creencias implícitas que dominan el quehacer del aula.

\section{Teorías implícitas y práctica pedagógica docente}

Cossío y Hernández (2016), definen las teorías implícitas como “(...) constelaciones complejas de conocimientos y creencias intuitivas, difíciles de explicitar, que se elaboran con fines pragmáticos de utilidad y que tienen como fuente de abastecimiento la suma de experiencias personales dentro de determinados contextos culturales" (p. 1139).

Estas creencias terminan por dominar la práctica profesional docente en distintas situaciones pedagógicas, sin atribuirlas a explicaciones formales sino a sus experiencias enmarcadas en un entorno cultural interpretativo y re interpretativo donde existen otras construcciones subjetivas semejantes que terminan por reforzar la explicación implícita de la persona.

A nuestro entender, las teorías implícitas se presentan como unas formas de pensamiento social identificadas como teorías espontáneas, intuitivas o ingenuas que subyacen en las cogniciones de los docentes acerca de la enseñanza y el aprendizaje. Como sistemas cognitivos complejos, no sucumben ni al tiempo ni a sus supuestas inconsistencias. De hecho, la resistencia al cambio es un aspecto por el cual las teorías implícitas destacan. Rodríguez y Gonzáles (1995), explican que "los individuos interpretan los eventos acudiendo a aquellas proposiciones causales de sus propias teorías (...)" (pp. 225-226).

Las teorías implícitas, como constructos primarios en la cognición humana, determinan muchas veces la dirección de la enseñanza en la práctica educativa, por lo que el circuito didáctico (propósitos, contenidos, estrategias, recursos y evaluación) se impregna desde lo que sabe por experiencia el docente que por lo que podría explicitarse en un modelo teórico, conceptual o técnico que pertenece al campo educativo. En efecto,

... "Las personas infrautilizan importantes fuentes de información tales como el tamaño de la muestra, la probabilidad previa de un suceso, etc. En su lugar emplean una serie de reglas y atajos (...) que aplican espontáneamente a los procesos de inferencia". (Kahneman y Tversky, 1972, 1973, citado por Rodríguez y González, 1995, p. 226)

Cuando nos referimos a las prácticas pedagógicas estamos en el ámbito de la actuación docente. En efecto, deben ser entendidas desde la perspectiva de las interacciones entre los docentes y los estudiantes en el aula, y que se relaciona con los logros de aprendizajes. Concretamente, es un conjunto de acciones "que ejecuta el maestro en el aula y que, implícita o explícitamente, promueven algún tipo de aprendizaje en sus estudiantes" (González y otros, 2017, p. 3).

Estas acciones, según Latorre (2004) pueden ser "experienciales, reproductivas y transformativas" (p. 88).

Las prácticas pedagógicas experienciales responden a estímulos internos y externos del estudiante, vinculan el sentido común y se disocia de toda norma. Se asocia a esta tipología los aprendizajes colaborativos. Las prácticas pedagógicas reproductivas responden a una línea de acción previamente normada por disposiciones que se generan en el ámbito institucional de la 
escuela para el logro de resultados inmediatos y efectistas. Sobresalen el desarrollo de respuestas cortas, la aplicación de exámenes, el uso de libros de texto escolar, etc. Por último, las prácticas pedagógicas transformativas adecúan la acción a la dinámica del contexto. Son estructuradas, flexibles y pertinentes, e incorporan los hechos a la situación. Comprenden aquellos proyectos de investigación que responden ante una situación problemática.

Cabe anotar que existe un vínculo entre las teorías implícitas y la práctica pedagógica que permite el funcionamiento pertinente del currículo escolar en su implementación. Un punto crucial en este proceso curricular es el estudio entre lo que piensa el docente y lo que realmente hace en sus clases. Frente a esta disyuntiva, los enfoques de las teorías de la acción asumen la existencia de dos tipos de sub teorías: aquellas,

(...) utilizadas por los profesores para explicar las razones de sus acciones, en las que se apoyan sus metas, intenciones y pensamientos (teorías asumidas); y las (...) que realmente determinan sus acciones y práctica (teorías en uso). (Soto y Barrios, 2011, p. 78)

Es decir, se configura una teoría adoptada o implícita que incluye la cosmovisión y los valores que el docente cree poseer para sustentar su comportamiento; a la vez, se presenta una teoría en uso que gobierna el mismo comportamiento del docente en situaciones reales. Comprender esta relación entre la teoría implícita y la práctica pedagógica confiere una explicación de mayor consistencia acerca de la implementación del currículo escolar en el aula y de cómo en ese proceso las resistencias subyacentes (voluntarias o involuntarias) de los docentes, dificulta la ruptura del desgastado paradigma tecnicista curricular.

En resumen, el problema de la implementación del currículo se sitúa en la reproducción de acciones pedagógicas desde una perspectiva instrumentalista, desvalorizando las relaciones intrínsecas entre el pensamiento docente y su actuar pedagógico; es decir, entre las teorías adoptadas y las teorías en uso. $\mathrm{Al}$ respecto:

Las teorías en uso (actuar pedagógico) son medios para obtener lo que queremos, pues especifican estrategias para resolver conflictos, organizar una clase, interactuar con el estudiante, etc. (...). En cambio, las teorías adoptadas (pensamiento implícito), se consideran como el contexto ideológico dentro del cual el docente percibe, interpreta, decide, actúa y valora. (Soto y Barrios, 2011, p. 78)

La implementación del currículo, según las orientaciones del Ministerio de Educación "implica la generación de condiciones, el análisis del Perfil de egreso, sus competencias, estándares, el contexto y las necesidades e intereses de los estudiantes (...)" (Ministerio de Educación, 2016, p. 8).

En este marco, la práctica docente, en cuanto al desarrollo del currículo, está amarrada a una idoneidad de perfil y competencias de logro, de estándares y desempeños declarados previamente, generando que la práctica pedagógica acentúe su pretensión para su cumplimiento. Sin embargo, en el actuar de los docentes no se llega a la comprensión de estas idoneidades porque aún las propuestas curriculares pertinentes no han sido diseñadas en las regiones y localidades para responder de manera pertinente a las problemáticas y necesidades particulares de las escuelas. En contraste con lo señalado en las orientaciones generales para la diversificación curricular que se menciona en el documento del Currículo Nacional (Ministerio de Educación, 2016, p. 210), donde se aclara que las competencias pueden contextualizarse sobre la realidad regional con base a estudios previos, según las características de los estudiantes y de sus entornos geográficos, económicos y socioculturales en cada realidad regional y local (...), o elaborarse nuevas competencias "pertinentes a las características específicas, necesidades y demandas de los estudiantes, en función de sus entornos socioculturales, lingüísticos, económico - productivos y geográficos", la realidad que 
se observa en las propuestas curriculares de la escuela y del aula, distan mucho de lo deseado en el documento oficial.

Finalmente, los docentes no logran asumir como propia la experiencia de adecuación de los propósitos de aprendizaje del currículo porque las disposiciones y procedimientos para la planificación y ejecución curricular, designados por el ente central, terminan por replicar el corpus tecnicista, sin que se consideren las maneras de pensar docente y su relación con la forma cómo realizan la concreción curricular en la realidad de sus escuelas.

\section{La racionalidad curricular dominante: el instrumento como prioridad}

Para Giroux (2004), la racionalidad técnica del currículo se concibe en términos de rigurosidad para el cumplimiento de objetivos concretos porque “(...) está vinculada a los principios de control y certeza. Su interés constitutivo de conocimiento reside en controlar el mundo ambiental objetivado" (p. 224).

De hecho, desde la perspectiva de la racionalidad técnica, la objetivización del conocimiento en un proceso de enseñanza aprendiza, se reduce a hechos y conceptos que son empíricamente comprobables. En un currículo aplicado desde dicha racionalidad, donde la verdad de una afirmación se convierte en lo relevante para un sistema de enseñanza, se "cumple los presupuestos de la racionalidad sí y solo sí encarna un saber fiable guardando relación con el mundo objetivo (...)" (Habermas, 1999, p. 26).

Por otro lado, Pascual (1998) señala que:

La racionalidad técnica tiene una correspondencia crucial con el desarrollo del paradigma conductista y los aportes teóricos al modelo pedagógico por objetivos, concebidos por Ralph Tyler. “(...) en esta noción bidimensional de objetivo educacional ya se incorpora esta racionalidad de adecuación de los medios a los fines, en cuanto la adquisición o modificación conductual del alumno constituye el fin y el medio (a través de) con los contenidos culturales (...)”. (p. 20)

Es decir, la perspectiva técnica curricular se propone "concretar el plan o el diseño previamente elaborado. Su éxito se mide en términos de la eficacia de sus realizaciones" (Feldman, 1999, p. 50). Cuando se observa la presencia de dicha perspectiva en el currículo escolar, se reconocen los medios que lo sostienen para asegurar su eficiencia y así generar el producto. Estos medios se constituyen en herramientas, procedimientos y tecnologías en general. De allí que el currículo escolar se convierta en un instrumento que se concreta en las diversas instancias de gestión hasta llegar al aula. A escalas mayores, la instrumentalización del currículo conlleva a la especialización de las necesidades profesionales que se expresan en documentos curriculares, paquetes instruccionales, propuestas didácticas, libros de textos, programas de mejora, modificaciones de la estructura escolar, etc. Por lo tanto, la racionalidad instrumental del currículo escolar plantea un aspecto crucial del problema en la implementación del mismo. Las prescripciones elaboradas por un grupo de expertos del ente central se orientan a cerrar todo alcance de reflexión de las prácticas implícitas de los docentes sin que puedan promover la construcción de propuestas curriculares de mayor pertinencia.

Por otra parte, Grundy (1998) propone ciertos criterios de reconocimiento de la racionalidad tecnicista en el currículo escolar. Señala que "cuando la acción está informada por el interés técnico (...) queda constituida por una serie de elementos. Se trata del eidos (la idea orientadora) y la tekné(la disposición orientadora) que, unidas, proporcionan la base de la poietiké ('hacer' la acción)" (p. 43). Precisando lo que sostiene el autor, el "eidos" (la idea) solo se concreta mediante la "tekné" (habilidad del docente). A su vez, el "eidos" prescribe la naturaleza del producto y no la habilidad del docente. Por tanto, el resultado de la "poietiké" (efectuar la acción) 
es algún producto. El producto será juzgado de acuerdo con la medida en que se parezca a la imagen implícita en el "eidos" orientador.

Los elementos descritos permiten identificar los rasgos tecnicistas del currículo escolar. A continuación, añadimos las precisiones que el documento señala sobre el currículo, en la página 8 , y realizamos un contraste con la propuesta de Grundy: el establecimiento de los aprendizajes es una prescripción que es justificado por los fines y principios educativos nacionales, lo que vendría a ser el "eidos". Luego, se propone el perfil del egreso del estudiante, que es el producto formado al finalizar la educación en el sistema educativo. Por último, se presenta la organización curricular por estándares, áreas, competencias, capacidades y desempeños, así como las orientaciones para la evaluación y diversificación curricular que se concretarían a través de la "tekné" y la "poietiké"; es decir, la habilidad y la acción pedagógica que juntas conforman la práctica docente.

La naturaleza tecnicista del currículo escolar también trae consecuencias en cuanto a la funcionalidad de su implementación, que, en muchos casos, terminan siendo disfuncionales en criterios de mejora de los aprendizajes.

En palabras de Pascual(1998), el currículo escolar prescrito es una normativa de política pública que implica formalizar "una oferta educativa o de estudios a seguir para los estudiantes, que es articulada en función de ciertos principios y valores socioculturales, necesidades humanas y sociales y políticas educativas" (p. 54).

Es así como se configuran dos formas curriculares: el currículo explícito, manifiesto o de producto, que se opone al currículo implícito, oculto o de proceso, centrado "en los contenidos de la interacción entre los actores educativos (profesores, alumnos, padres, dirección, etc.) en la acción pedagógica misma de la unidad escolar" (p. 55).

Estas concepciones de currículos conviven en la cultura escolar, y la práctica pedagógica se sitúa entre ambas formas, sin que los docentes hayan podido articularlas. Además, las dos formas de concepciones entran en disonancia cuando los docentes "se limitan a aplicar las prescripciones de un diseño curricular o de un plan de acción educativa, sin reflexión crítica, ni asimilación y/o negociación o consenso entre ellos (...)” (p. 56).

En cuanto a la perspectiva del currículo como producto, ella genera un divorcio entre el diseñador (especialista de alto nivel) y el ejecutor (docente). Es decir,

...se separa el proceso de su construcción, por expertos de la práctica curricular misma en la escuela, gestionada por los profesores (...). Y cuanto más se aleja, se corre el riesgo de que el diseño curricular se convierta en una prescripción vacía, meramente formal (...). (Pascual, 1998, p. 59)

Esta separación conduce a limitar la construcción reflexiva de la práctica pedagógica y trae como consecuencia la desarticulación entre las necesidades educativas de los estudiantes y el contexto en el cual se encuentra inmersa la escuela. Muchas veces el currículo explícito se ve comprometido cuando se condice con la realidad en los procesos de aprendizaje y enseñanza.

Otro aspecto que también es importante para el entendimiento de la brecha curricular entre lo formal y lo real, se relaciona con el análisis del sistema de la evaluación de los aprendizajes como componente clave en un sistema curricular. La experiencia en evaluación educativa en todos los niveles ha tenido avances significativos en las mediciones de los aprendizajes desde la aplicación de pruebas estandarizadas con fines de rendición de cuentas. Sin embargo, la evaluación de los resultados como parte de la implementación del currículo en las escuelas no han sido claras, menos innovadoras. Se supone que las escuelas, antes que precisen el proceso sumativo de sus avances, deben incluir procesos más abiertos, flexibles y comprensivos con fines de mejora, rasgos fundamentales 
de la evaluación formativa. Además, la instrumentalización que construye el sistema desde arriba y las creencias implícitas de los docentes para reproducir lo que han venido realizando en años con el método de exámenes, con preguntas cerradas o abiertas, y con ítems orientados al desarrollo de habilidades de orden inferior, terminan por dificultar las miradas reflexivas sobre los resultados.

Por consiguiente, para el caso del currículo escolar, tanto el sistema educativo como los docentes creen de manera arraigada que la medición de los aprendizajes deben priorizar el conocimiento del contenido hacia el aprendizaje de habilidades de niveles reproductivo, comprensivo y analítico, que incluyen el aprendizaje de formulismos, operaciones, definiciones, conceptos, características, elementos, nombres técnicos, entre otros elementos, desvirtuando la mejora del sistema y reforzando la eficiencia del sistema curricular.

\section{CONCLUSIONES}

La propuesta del currículo escolar en el Perú ha tenido ciertos avances, a pesar de las diversas reconceptualizaciones que generaron la revisión de sus elementos conceptuales y operativos. Sin embargo, el contraste ha sido evidente porque la racionalidad dominante que se presenta en el currículo escolar y en las prácticas docentes se traduce en aquella concepción técnica, objetivista y cerrada que tendrá un costo en el desaprender. Asimismo, las creencias implícitas de los docentes se mantienen intactas porque el sistema gestiona todos los componentes de especialización que comprenden el currículo como las normativas, programas, jornadas de capacitación, textos escolares, instructivos de implementación, la estructura misma de un perfil de egreso, enfoques transversales, estándares y desempeños; las unidades y sesiones de manera general como modelos de orientación, etc. Estos elementos, limitan el desarrollo de la discusión en cada realidad que corresponde a cada escuela, diversa en sus interacciones y manifestaciones socioculturales.

Por otra parte, el riesgo que el currículo escolar tendrá que enfrentar es su propia normativa de formalización que encuentra su propia dialéctica en la realidad del aula y la experiencia del docente. Lo que se observa, a partir de las diversas manifestaciones de los docentes es que no terminan de comprender las razones y propósitos del currículo formal, y por la demanda del tratamiento inmediato del currículo en los procesos de planificación y ejecución durante el año, se enfrascan en la rutina a inicios del año, sin haber generado espacios de reflexión acerca de las prácticas pedagógicas y las creencias que aún mantienen los docentes con respecto a la educación.

Por último, la perspectiva instrumentalista del currículo también influye en las prácticas de la evaluación de los aprendizajes. El currículo escolar ya ha prescrito los estándares, capacidades y desempeños como los criterios de evaluación previamente establecidos, y los procesos de aprendizaje y enseñanza se orientan al cumplimiento de estos enunciados que deben responder a una agenda estratégica regional de mejora de los sistemas educativos insertados en macropolíticas diseñadas por entidades multilaterales. 


\section{REFERENCIAS}

Cossío, E. y Hernández, G. (2016). Las teorías implícitas de enseñanza y aprendizaje de profesores de primaria y sus prácticas docentes. Revista Mexicana de Investigación Educativa, 21(71), pp. 1135-1164. Recuperado de: http://www. comie.org.mx/revista/v2018/rmie/index. php/nrmie/article/view/105/105

D'Amico, M. V. (2016). La definición de la desigualdad en las agendas recientes de los organismos internacionales para América Latina. Revista Colombiana de Sociología, 39(1), pp. 221-240. doi: https://doi.org/10.15446/rcs.v39n1.56348

Feldman, D. (1999). Ayudar a enseñar. Relaciones entre didáctica y enseñanza. Buenos Aires, Argentina: AIQUE.

Giroux, H. (2004). Teoría y resistencia en educación. Una pedagogía para la oposición. México, Ciudad de México: Siglo XXI.

Gonzáles, W. (2016). Las teorías implícitas de docentes acerca del currículo escolar: lo "oculto" en la escuela. Revista Tarea. Desafíos del Currículo Nacional de la Educación Básica (92), pp. 43-48. Recuperado de: https://tarea.org.pe/wpcontent/uploads/2016/12/Tarea92_43_Mario_Wilfredo_Gonzales.pdf

González, N., Eguren, M. y De Belaúnde, C. (2017). Desde el aula: una aproximación a las prácticas pedagógicas del maestro peruano. Documento de trabajo $\mathrm{N}^{\circ}$ 233. Lima, Perú: Instituto de Estudios Peruanos.

Grundy, S. (1998). Producto o praxis del currículum. Madrid, España: Morata.

Habermas, J. (1999). Teoría de la acción comunicativa I. Madrid, España: Taurus.

Latorre, M. (2004). Aportes para el análisis de las racionalidades presentes en las prácticas pedagógicas. Estudios Pedagógicos, 30, pp. 75-91. Recuperado de: http://revistas. uach.cl/pdf/estped/n30/art05.pdf

Ministerio de Educación (2016). Currículo Nacional de la Educación Básica. Lima, Perú. Recuperado de: http://www.minedu. gob.pe/curriculo/pdf/curriculo-nacionalde-la-educacion-basica.pdf

Pascual, E. (2011). Racionalidades en la producción curricular y el proyecto curricular. Pensamiento Educativo. Revista de Investigación Educacional Latinoamericana, 23(2), pp. 13-72. Recuperado de: https:// pensamientoeducativo.uc.cl/index.php/ pel/article/view/129

Pozo, J., Scheuer, N., Mateos, M. y Pérez, P. (2006). Las teorías implícitas sobre el aprendizaje y la enseñanza. En J. Pozo et al. (Eds.), Nuevas formas de pensar la enseñanza y el aprendizaje, pp. 95-132. Barcelona, España: Grao.

Resolución Viceministerial Nº 024-2019-MINEDU (2019). Orientaciones para la implementación del Currículo Nacional de la Educación Básica en el año 2018. Lima, Perú: Ministerio de Educación.

Rodríguez, A. y González, R. (1995). Cinco hipótesis sobre las teorías implícitas. Revista de Psicología General y Aplicada, 48(3), pp. 221-229. Recuperado de: https://dialnet.unirioja.es/servlet/ articulo? codigo $=2161352$

Soto, D. y Barrios, M. (2011). El Pensamiento de los profesores, sus teorías implícitas y la relación con la práctica, un estudio de caso de los profesores de la Universidad Pedagógica Nacional. Tesis de pregrado. Recuperado de: http:// digitalacademico.ajusco.upn.mx:8080/ tesis/handle/123456789/9126 
106 | unife 